No. 4004 July 27,1946

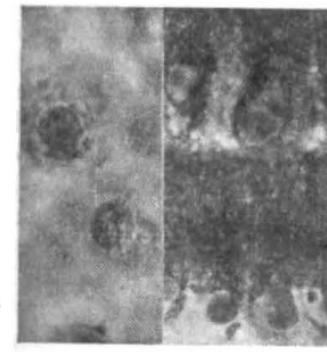

$a$

CELLS FROM EMBRYOS OF BARLEY SHOWING DIFFERENCES IN NDCLEOLI AND STAINING INTENSITY IN NORMAL GRAIN (a), HEAT-DAMAGED GRAIN (b) AND HEAT-KILLED GRAIN $(c)$, AFTER 24 HR. INCUBATION AT $27^{\circ} \mathrm{C}$.

(2) Heat-damaged living grain showed at first no change in the nucleolus. The duration of this quiescent period depended on the severity of the heat treatment. Then activity as in normal grain occurred, but as the nucleolus increased in size it yielded blister-like protrusions (Fig. b). This activity was accompanied by a slower decrease in the basophily of the cytoplasm. When serious blister formation occurs, the damage is apparently irreversible.

(3) In heat-killed grain there was no change in nucleolar size; the basophily of the cytoplasm showed no decrease and may even have increased slightly (Fig. $c$ ).

The observations on the nucleolus suggest that, even when still alive, the damaged cell has lost some of its power of selective permeability. It is unable when active to control the entry of water into the nucleolus. Support for this suggestion is obtained from the fact that when water uptake is restricted, the abnormal nucleolar behaviour in damaged grain is much less marked, and the grains eventually germinate. Nevertheless, before germination damaged grain still shows the lag in development of the nucleolus. The rate of decrease in basophily of the cytoplasm to the level at germination, and later, is similarly slower. The sensitivity of damaged grain to excess water and to the formation of nucleolar protrusions thus seems to be secondary and due to alterations in permeability.

The primary factor in heat damage seems to be an inactivation of the cell, accompanied by an alteration in permeability which may be of much less importance. As the chemical activity of the cell is believed to be essentially enzymic in character, such inactivation suggests at least one vital heat-labile system in the earliest stages of the chain of activity preceding cell division, and such a bottleneck may be responsible for the delay in germination. This delay may well be due to inactivation of enzymes responsible for reduetion of ribose nucleic to desoxyribose nucleic acid. The changes in staining intensity would agree with such an interpretation.

Measurements of carbon dioxide output of respiring grain $^{5}$ indicated that the respiration of mildly damaged grain was almost normal, and even of severely damaged grain was much greater than corresponded to its final germination capacity. Thus such figures were quite useless as tests for mild heat damage, and even grain incapable of germination showed a carbon dioxide production one fifth or so of that of normal grain (at 30 per cent moisture content). The respiration death point (that is, where respiration was but 1-2 per cent of normal) was some $4^{\circ} \mathrm{C}$. above the germination death point. It thus seems that mild heat damage is not accompanied by, and could not be attributed to, inactivation of enzymes primarily concerned with the respiration process. Similarly with animal tissue Peters reports that after heat treatment at the marginal damage temperatures, tissue respiration showed no fall.

Quoting Peters' report: “' $50-55^{\circ} \mathrm{C}$. for $30-60$ seconds is a critical temperature for damage, producing permeability changes with liberation of substances from the skin cells, which can be detected histologically. The degree of damage has been correlated with increasing temperature. Higher temperatures induce a different condition, including fixation and coagulation of the protein; the actual temperature for damage is $48-50^{\circ} \mathrm{C}$. This tempera. ture can destroy some enzymes in a short period; but it is not yet decided whether enzyme damage or change in lipoprotein in the cell interface is the initial biochemical alteration."

Dry wheat at 14 per cent moisture content is not strictly comparable with living tissue, because of the influence of water content upon temperatures of damage, but grain at 37 per cent moisture content, as just prior to germination, if immersed in hot water for one minute, shows a damage zone of $53-62^{\circ} \mathrm{C}$. (water temperatures). The results of Hutchinson and Booth indicate that at the death temperatures of the wheat grain there is severe protein coagulation and enzyme inactivation, and that in damp grain treated at the low temperature of damage which causes but little delay in germination, alterations can be detected in the grain which are almost certainly due either to a form of protein coagulation or to enzyme inactivation. Careful observation reveals some slight germination delay at temperatures a few degrees below $53^{\circ} \mathrm{C}$., and indicates too that vitality as judged by ability to withstand excess water and other sub-optimal germination conditions is probably adversely affected. It may thus be that in the case of grain, as with animal tissue, the first biochemical alteration is a permeability change, whereas the retardation of germination is probably caused by enzyme inactivation at slightly higher temperatures.

"Peters, R. A., "The Biochemical Lesion in Thermal Burns", Brit. Med. Bull., 8, 81 (1945).

${ }^{2}$ Hutchinson, J. B., J. Soc. Chem. Ind., 63, 104 (1944).

${ }^{3}$ Peto, F. H., Can. J. Res., 9, 261 (1933); 13C, 301 (1935) ; 15C, 217 (1937).

4 Darlington, C. D., Discovery, 6, No. 3, 79 (1945).

'Greer, E. N., and Hutchinson, J. B. (unpublished).

- Hutchinson, J. B., and Booth, R. G., J. Soc. Chem. Ind. (1946), in the press.

\section{DIELECTRICS IN THEORY AND} APPLICATION

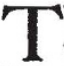
TW discusfins on the subject of dielectrics were gelde ferently, the first by the London branches of tho Royal Institute of Chemistry and the Instity o of Pysies jointly at the Royal Institution on M 1 re 20 , the second by the Faraday Society during 4 pril 24-26 at the Wills Physical Institute at Brifil. The former meeting surveyed broadly the field of physical theory, chemical preparation and industrial application of the subject, while the latter discussed surveys and original papers on the present state and immediate trends of physical chemical and physical research.

Dealing first with the more 'industrial' side, N. J. L. Megson, adviser on plastics to the Ministry of Supply, showed that the most important new dielectrics are 
usually of the high-polymer type. Most of the latter are either 'thermoplastic' (plastic when hot) materials with long chains having considerable freedom of movement, or 'thermosetting' (hardened by heat) materials which when heated yield a cross-linked structure with little freedom. Either type may be produced by condensation or polymerization. The general type of electrical properties can be predicted from the chemical structure; for example, the improvement from the 'Novolak' phenol,formaldehyde to the cresol- or xylenol-formaldehyde and thence to the benzyl resins may be ascribed to the relative increase of hydrocarbon and decrease of polar hydroxyl groups. The polar amino structure of urea and similar resins gives electrical properties no better than those of phenolic resins; but the high nitrogen content may be the reason why they are less subject to 'tracking'. Polymerized hydrocarbons such as polythene and polystyrene are excellent dielectrics, but are softer and less resistant to heat due to the absence of cross-linkages. Some increased resistance to heat has been achieved by substituting chlorine for hydrogen in polystyrene. Another recent method of inducing heat resistanee is by the polymerization of doubly unsaturated compounds, such as the diallyl derivatives, from which resins are obtained resembling the methyl-methacrylate resins but having thermosetting characteristics. Lastly, there is the substitution of earbon by silicon obtained in the 'silicones', which are based on the $-\mathrm{O}-\mathrm{Si}-\mathrm{O}-\mathrm{Si}-$ chain and can be cross-linked. Although these last are highly resistant to heat, and the other methods have also shown promise, the plastic, which is an excellent dielectric, resistant to heat and easily utilizable, is not yet in production.

A. J. Maddock dealt with dielectric heating where the materials involved, such as glues, wood and fabric, are not always regarded as dielectrics. Heating by dielectric losses in a high-frequency field has the advantages that the heat is generated uniformly throughout the volume where it is wanted, and the low thermal inertia permits rapid heating and close control. Frequency is not critical, since the total energy loss as the frequency passes through that of a dipolar absorption shows no maximum and the losses in practice change only slowly. While the idea was suggested by von Siemens and Tesla, it was not until 1935 that Leduc applied the method to vulcanizing rubber, while the major development has been confined to the past few years. In the range 2-5 Mc./s., powers up to hundreds of kilowatts are used; from 10 to $25 \mathrm{Mc} / \mathrm{s}$. medium powers apply with instances up to $100 \mathrm{~kW}$.; from 50 to $80 \mathrm{Mc} / \mathrm{s}$. the power does not usually exceed $1 \mathrm{~kW}$.; while in the range $100-200 \mathrm{Mc} / \mathrm{s}$. only low-power special applications are involved.

A very successful application is the preheating of plastic materials prior to moulding and the polymerization of thermosetting resins used to bond paper, fabric and wood into boards or articles such as gear wheels. In bending plywood in two directions the material can be built up over the former by lightly 'tacking' it with a species of 'gun': the assembly is then compressed and heated in its final shape, since the 'tacks' can then break. The gun is formed of a coaxial transmission line, the open end of which is pressed on to the work, by which the high-frequency field is applied locally in the vicinity of the end. The combination of this analogue of spot-welding with shaped electrodes for seams enables plastic, bonded and glued articles to be made by rapid and continuous processes usually associated with more amenable materials such as fabrics without the damage involved in operations such as stitching. Dielectric heating may be used for drying provided the rate of extraction of moisture is not a limiting factor; an example is in drying artificial silk. Possibilities being explored lie in reheating cooked foods, softening frozen foods and the sterilization of poor thermal conductors.

The present state of the physical theory of solid dielectrics was surveyed by $S$. Whitehead, director of the British Electrical and Allied Industries Research Association, at the London meeting, and by H. Fröhlich (to whom recent theoretical developments are largely due) at the Bristol meeting in rather similar terms. The absorption bands or natural frequencies of small molecules lie in the visible or near infra-red; but with large molecules, such as are encountered in dielectrics, the absorption bands can be of much lower frequency. These collision-broadened bands can influence the electromagnetic spectrum, for example, at $10^{10}$ cycles per sec., because Van Vleck and Weisskopf and also Fröhlich have shown the classical Lorentz theory to give too small an effect at frequencies remote from the natural frequency. The normal electromagnetic range is influenced by dipoles which, in many solids as opposed to liquids, may only have one direction with respect to the molecular axis, but of either sense in that direction. When the dipolar interaction is small, as in dilute solutions or at high temperatures, the transition of the dipoles from one direction to the opposite provides a relaxation time and a resultant variation of dielectric constant and loss angle very similar in shape to that derived from the Debye theory, except for the values and significance of the parameters. When most of the molecules are dipolar so that their interaction is important, and when the temperatures are low, the turning of some dipoles may facilitate the turning of others in the vicinity, leading to an effect analogous to the 'domain' hypothesis in ferro-magnetism.

The transition from the low-temperature to the high-temperature case occurs at a critical temperature which may be the melting point, and is of the type known as a $\lambda$-transition, where the dielectrio constant can reach very high values and where there is a discontinuity in the specific heat, in conformity with the work of Müller and Ubbelohde. In the hightemperature or dilute-dipole region, it seems that the right shape of dielectric constant and loss-angle curves is probably only found with ice, so that with this exception supplementary hypotheses such as those of Yager and of Garton of a distribution of relaxation times are required to explain experimental results on solids. Lamb confirmed at Bristol the behaviour of ice except that a large power-factor at $10^{10}$ cycles per sec. requires an additional source of absorption; while a paper by Smyth, in essence, approached some of the foregoing hypotheses from the aspect of molecular freedom.

Some detailed discussion centred around the theory of the internal field in dielectrics. Debye, Onsager and Fröhlich all regard the dielectric as a continuum with respect to a dipole; by the first, the medium is supposed to react slowly to change of dipole orientation; Onsager thinks the medium reacts quickly compared with the dipole; while Fröhlich takes into account the relative magnitudes of the relaxation times of dipole and medium, indicating that Onsager should be right at high 
temperatures. Actually, however, the vicinity of a dipole has a 'discrete' structure, and Sack has calculated the exact effect of short-range interaction for a single-dimensional case of a chain molecule, showing that the frequency dependence according to Debye or Fröhlich is unaltered. Baur and Massignon put forward a calculation of the interaction potential in a crystalline field, or rather a general method of calculation. Frank concluded in general that Debye's 'hindering energy' has real significance and that Onsager must always be to some extent in error, while he also gave an analysis of certain detailed types of interaction. Kirkwood and also Böttcher considered the internal field in an amorphous fluid; the former gave an exact theory for the sphere of influence around a dipole considered as fixed.

Dipole relaxation is generally associated with a species of viscosity in liquids, but in solids is held to be a thermally activated transition across a potential barrier; although Pelzer directed attention to Kramer's view that a kind of diffusion analogous to Brownian motion forms a more general concept to which the activation process is an approximation. But the differences discussed between experiment and existing theory may well have been greater than possible errors on this account. Fröhlich has explained that the relaxation time in a chain molecule should increase at first proportionately with chain-length but thereafter more slowly with an asymptotic curve. The papers by Willis Jackson and Powles on benzene and paraffin solutions, Collie, Ritson and Hasted on water, Abadie on acetone and water, Whiffen and Thompson on pure hydrocarbons, related materials and solutions, supported the general outline of fairly well-defined relaxation times, but no simple concept of viscosity can be applied. Nevertheless, particular interest is attached to the first paper, because it is believed that the experiments on benzene solutions are the first to show a good agreement with Debye's theory with a single relaxation time, due possibly to the small molecules involved.

Girard and Abadie indicated the wide deviations from theory which are observed in some cases, and mentioned the complexity introduced if the liquid molecules were conceived as ellipsoidal instead of spherical; Schallamach has studied the properties of mixtures of dipolar liquids and the multiple absorption bands which can occur. As to solids, Oakes and Richards in the case of chlorinated polythenes, Plessner and Richards in the case of solutions of esters in polyisobutylene and polythene, Carter and others in the case of natural and synthetic rubbers, have applied with some success the hypothesis of Fuoss and Kirkwood, which is essentially similar to Garton's earlier theory already mentioned of a distribution of relaxation times. However, Garton and also Gevers and du Pré addressed themselves to the problem of good dielectrics with small power factors which vary only slightly with frequency over wide ranges. Both papers adopted the Pelzer-Wigner transition-rate method with a distribution of heights of the potential barriers. Garton considers the transition from permanent 'potential wells' or equilibria to temporary 'wells', the depth or binding energy of which have a Maxwellian distribution, whence the relative constaney of the power factor follows. Gevers and du Pré, however, only go so far as to indicate the general kind of distribution of potential barriers which can explain observation, ignoring Garton's point that the polarizability depends on the relative life of a dipole in a given state.
This modification of the rate process stimulated discussion, as also did the attempts to identify the potential barriers by Eyring's method with activation energies and entropy changes associated with physical-chemical bonds, particularly when, as in Whiffen and Thompson's work, the entropy change of activation appeared negative. It was shown that this anomaly is entirely dependent on the multiplying factor in Eyring's equation which, so to speak, sets the time-scale, so that it is difficult, if not impossible, to make an absolute interpretation on these lines in absence of an exact solution of the particular solid structure, although relative changes should be valid. A relative change is involved, for example, in the comparison of homologues with different chainlengths, where Fröhlich's views seemed qualitatively to be confirmed. Lastly, Whitehead directed attention to the use of linear theory in analysing dielectric properties, such as eliminating undesirable or inaccurate parameters, and the difficulty attending experimental verification unless the crucial range can be properly chosen.

Perovskite crystal types which include the high dielectric constant titanates formed the subject of two papers. The structure is that of connected octahedra as regards the oxygen atoms, or cubic as regards the titanium (or similar element) atoms, with a central large metallic cation. According to Miss Megaw, if the latter is too small for stability, the cubic symmetry may be distorted to monoclinic form; but in some compounds, including those with high dielectric constants, the metallic cation is too large and there is tetragonal distortion with a $\lambda$ transition to the cubic form above a critical temperature, with possibly another reverse $\lambda$ transition at a lower temperature analogous to the double transition in piezoelectric crystals. Rushman and Strivens, adopting this picture, considered the possibility of the small titanium ion's 'rattling' in the structure distorted in the tetragonal manner, so that an effective dipole is produced. They claimed that the general behaviour of these crystals could thus be explained, and the high dielectric constant would be an example of the $\lambda$ transition and an analogue to the ferro-magnetism discussed by Fröhlich. However, the highest dielectric constant does not actually occur at the temperature for the crystalline transition, and in the discussion doubt was cast on the hypothesis that the polarization was due to the type of ion displacement described.

A notable feature of the papers at Bristol was the enormous advance made during the War in highfrequency dielectric measurements in the centimetric wave-lengths, up to $2.5 \times 10^{10} \mathrm{c} / \mathrm{s}$. The infra-red region is not very remote from this, and some discussion took place on the use by Whiffen and Thompson of what is essentially an infra-red technique adapted to these frequencies. Willis Jackson summarized the main features of resonance and standing-wave methods applied particularly to coaxial lines and wave guides; he gave the basic relations between complex dielectric constant, refractive index, power factor, permittivity, propagation constant, attenuation coefficient and phase constant, indicating the way in which these various but equivalent parameters appear in different methods of measurement and description.

At the London meeting, Megson, and at Bristol, Sutton, indicated the many ways in which dielectric studies have elaborated or given concrete expression to chemical concepts. The importance in engineering 
is daily self-evident. The theoretical physicist using the dielectric approach has probably made a more remarkable contribution to the theory of the solid state and, to a less extent, the liquid state; but his fertility has rather overwhelmed the experimentalist. The rich and varied experimental techniques disclosed at these meetings give, however, high promise for the eventual elucidation of these difficult but fundamental problems.

\section{OBITUARY}

\section{Sir George Julius}

WHEN the Australiay Council for Scientific and Industrial Regh $h$ was established in 1926, Sir (then Mr.) George (x is was invited to take the part-time chairmang the Council and of its Executive Commit. U. He held office until the end of 1945, and o was ixtimately associated with all the developments of this virile organisation during its first twenty fears. He died on June 28 at the age of seventy hree.

Born in Norwich, Julius was educated at the Church of England Grammar School in Melbourne and later at the University of New Zealand, where he attended classes with his contemporary and friend, Ernest Rutherford. Graduating in engineering, he was appointed in 1896 to a post in the Locomotive Department of the Western Australian Railways, and while there he carried out an extensive investigation of the mechanical properties of Australian hardwoods. After eleven years he left Government service and set up as a consulting engineer in Sydney. His firm (later Julius, Poole and Gibson) was in the course of years associated with very many of the main engineering enterprises of Australia.

Sir George's personal contacts were wide, but his chief interest lay always in mechanical invention, much of his work being done in a very fine private workshop at his beautiful home at Darling Point on the edge of Sydney Harbour. $\mathrm{He}_{\Theta}$ is perhaps best known to most people for his work on the Julius totalizator, now in use on many racecourses throughout the world. His father, the late Churchill Julius, formerly Archbishop and Primate of New Zealand, and himself no mean amateur engineer, was always deeply interested in his son's inventions, including all improvements to the totalizator; but he was careful to preface an approach to the subject by a declaration that such machines might well be sunk in the Harbour!

In 1898 Julius married Eva, the third daughter of another very famous Australian engineer, Mr. C. Y. O'Connor, who built the Mundaring Weir at Perth and, despite deplorable political interference, carried through to complete success a scheme for the transmission of water from Mundaring to the goldfields of Kalgoorlie and Coolgardie.

As president of such bodies as the Australian Institution of Engineers, the Engineering Association of New South Wales, and the Electrical Association of New South Wales, Sir George played a notable part in the professional public life of his adopted country. He was knighted in 1929 and later received the honorary degree of D.Sc. from the University of New Zealand. His last visit to England was in 1927, when he attended the Empire Agricultural Conference which led to the establishment of the Imperial Agricultural Bureaux. David RrvetT

\section{NEWS and VIEWS}

\section{5. \\ New President of the British Association :}

Sir Henry Dale, O.M., G.B.E., F.R.S. Sir HenRY ' Hue has been elected president for the year 194 of the British Association for the Advancenfy of Seience. Sir Henry was formerly directory the National Institute for Medical Repcl and for ten years before that he directed th Whllcome Physiological Research Laboratories $(19,14)$. He has achieved world recognition for his work on the physiological effects of histamine, a derivative of ergot, which was followed by brilliant researches resulting in the isolation of histamine and acetylcholine from animal tissues. Later work was devoted to examining the part played by these substances in physiological and pathological processes. Many other research workers have carried out their investigations under Sir Henry's guidance.

But apart from his own scientific research work, Sir Henry Dale has taken a very active part in the organisation of scientific work in Great Britain. For ten years (1925-35) he was one of the secretaries of the Royal Society of London and in 1940 was elected president of the Society-an office which he filled with conspicuous success until his statutory resignation in 1940 . Until September of this year he is director of the laboratories and Fullerian professor of the Royal Institution-a post he has held since 1942. He is also a member of the Medical Research Council. He is a foreign member of numerous learned societies throughout the world. It is clear that in view of Sir Henry's outstanding career both as a scientific research worker and as administrator and organiser, the Council of the British Association has made an eminently wise choice for its next president.

Botany at the Fouad I University, Cairo

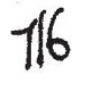

Prof. F. J. Lewis

Prof. F. J. Lwis has recently retired from the chair of botply in the Fouad I University, Cairo, which he hy held since 1935. A graduate of the University of Liverpool, he devoted himself to ecologica nvestigations and published a number of ingporlant papers on the post-glacial beds of the pest plosses of the north of England and also of Sfotland. He gave up his lectureship in Liverpool to beome professor in the newly founded university of Alberta, where he continued his ecological work, relating it to the rigorous climatic conditions of Canada. It must have been a great change for him to settle in Egypt; but new opportunities for ecological investigations presented themselves and led him to the establishment of a desert laboratory some little distance from Cairo. Very different problems also presented themselves by the accumula. 